\title{
NEW ESTIMATES FOR THE COLLAPSE OF THE MILNOR-MOORE SPECTRAL SEQUENCE OVER A FIELD
}

\author{
BARRY JESSUP
}

(Communicated by Frederick R. Cohen)

\begin{abstract}
We use the free tensor models of Halperin and Lemaire to give a new and transparent proof of a theorem of Ginsburg's on the collapse of the Milnor-Moore spectral under the assumption of finite L.-S. category. The method, valid over any field, provides better bounds for the collapse and these bounds are effectively computable L.-S. type invariants.
\end{abstract}

\section{INTRODUCTION}

A well-known result due originally to Ginsburg [Gi] states that if the LusternikSchnirelmann category $\operatorname{cat}(S)$ of a space $S$ is $m$ then the Milnor-Moore spectral sequence (hereafter $M-M$ s.s) for $S$ converging to $H(S)$ from $\operatorname{Ext}_{H *(\Omega S)}(k, k)$ collapses at the $(m+1)$ st stage. Ginsburg's was an intricate argument and later, Ganea [G] used his new characterization of $L-S$ category to present another proof that simplified somewhat the difficult step in Ginsburg's proof, namely, the effect of finite category on a geometric analogue of the bar construction due to G. W. Whitehead.

Halperin and Lemaire have recently defined free tensor models [HL] and used them to define new $L-S$ type homotopy invariants $\operatorname{Mcat}(S ; k)$ and $\operatorname{Acat}(S ; k)$ over any field $k$. These are much easier to compute than the classical cat $(S)$ and provide lower bounds for it when $S$ is simply connected. They can, for example, be computed fairly readily from an Adams-Hilton model for $C *(\Omega S)$, while $\operatorname{cat}(S)$ is usually difficult to calculate.

We use these free tensor models to prove the

Theorem. Suppose $S$ has the homotopy type of a simply connected $C W$ complex of finite $k$-type. If $m=\operatorname{Mcat}(S ; k)$, then the Milnor-Moore spectral sequence for $S$ collapses at the $(m+1)$ st stage.

Note that this result is sharper than the classical theorem in the case

Received by the editors March 15, 1990 .

1991 Mathematics Subject Classification. Primary 55M30, 55P60, 55P62.

Key words and phrases. Milnor Moore spectral sequence, Lusternik-Schnirelmann category, free tensor model.

This work was partially supported by the National Science and Engineering Research Council of Canada. 
$S=\operatorname{Sp}(2)$, where $\operatorname{Acat}(S, k)=2$ and $\operatorname{cat}(S)=3$. See [HL, S] for details.

\section{Notation AND Definition of $\operatorname{Mcat}(S ; k)$}

We shall work with the cohomology $M-M$ s.s., which we now briefly recall. Let $k$ be any field, and let $\left(C^{*}(S ; k) d\right)$ denote the graded differential algebra of $k$-valued singular cochains on $S$, augmented by a chosen nondegenerate base point. If $B\left(C^{*}(S)\right)$ denotes the reduced bar construction on $C^{*}(S)$, we can then apply the Adams cobar construction to this and the result is the tensor algebra on $B^{+}\left(C^{*}(S)\right)$, the canonical complement to $k$. The tensor powers $T^{\geq k}\left(B^{+}\left(C^{*}(S)\right)\right.$ are stable under the differential and upon filtering by them we obtain the $M-M$ cohomology spectral sequence $\left(E_{r}, d_{r}\right)$, where $E_{2} \cong \operatorname{Ext}_{H_{*}(\Omega S ; k)}(k, k)$ and which converges to $H^{*}(S ; k)[\mathrm{FH}, \S 9]$.

We need another, equivalent description. Let $(T(V), d) \stackrel{\cong}{\rightrightarrows}\left(C^{*}(S, k), d\right)$ be a minimal free tensor model for the simply connected space $S$. Then $V=$ $\bigoplus V_{j}, j \geq 2, V$ is of finite type and the differential $d$ increases "tensor degree" by at least one: $\quad \stackrel{d}{\rightarrow} T^{\geq 2}(V)$. Here, as above, $T^{k} \mid !(V)$ denotes $V \otimes \cdots \otimes V$ ( $k$ times). We bigrade $T(V)$ by setting $T(V)^{p, q}=\left[T^{p}(V)\right]^{p+q}$ where $p+q$ is the total (topological) degree. Above, the " $\simeq$ " means that the morphism of differential algebras is a quism, i.e., it induces an isomorphism on cohomology.

Filtering $(T(V), d)$ by the ideal $\left(T^{\geq k}(V), d\right)$, we obtain a naively convergent spectral sequence $\left(E_{r}, d_{r}\right)$ which coincides with the classical cohomology $M-M$ s.s defined above from the $E_{l}$ term on [H-L, Proposition 1.6]. From now on we refer to either as the $M-M$ s.s.

Now let $(T(V), d) \stackrel{j}{\rightarrow}\left(T\left(V \oplus V_{m}\right), d^{\prime}\right) \stackrel{\widetilde{\rightrightarrows}}{(}\left(T(V) / T^{>m}(V), \bar{d}\right)$ be a free model for the projection $(T(V), d) \stackrel{\pi}{\rightarrow}\left(T(V) / T^{>m}(V), \bar{d}\right)$. Then $\operatorname{Mcat}(S ; k)$ is defined as the least integer $m$ for which there is a retraction of $T(V)$-differential modules $\left(T\left(V \oplus V_{m}\right), d^{\prime}\right) \stackrel{r}{\rightarrow}(T(V), d)$, i.e., $r$ satisfies $r \circ j=\mathrm{id}$. (To define $\operatorname{Acat}(S ; k)$, we ask that $r$ be a map of algebras.)

The key ingredient of the proof of our theorem is a beautifully explicit model $(T(V) \otimes(k \oplus M), D) \stackrel{\psi}{\rightarrow}\left(T(V) / T^{>m}(V), d\right)$ in [FHLT], which we describe. The bigrading of $T(V)$ above can be carried to $T(V) / T^{>m}(V)$ by making $\pi$ homogeneous of bidegree $(0,0)$. Let $M=M^{m, *}=s T^{m+1}(V)$, i.e., $M^{m, q}=s\left[T(V)^{m+1}, q\right]$. There is an isomorphism $\omega: T(V) \otimes M \cong T^{>m}(V)$ defined by $\Omega(\Phi \otimes s \Psi)=(-1)^{|\Phi|} \mid \Phi \cdot \Psi$ where $|\Phi|$ is the topological degree of $\Phi$. We can then define a differential $D$ on $T(V) \otimes(k \oplus M)$ by $D=d$ on $T(V) \otimes 1$ and $D=\omega-\omega^{-1} d \omega$ on $T(V) \otimes M$. This makes $(T(V) \otimes(k \oplus M)$, $D)$ a differential $T(V)$-module, and an easy calculation shows that the map

$$
(T(V) \otimes(k \oplus M), D) \stackrel{\Psi}{\rightarrow}\left(T(V) / T^{>m}(V), \bar{d}\right)
$$

defined by $\Psi(v)=\pi(v), \Psi(T(V) \otimes M)=0$, is a quism.

We can filter $(T(V) \otimes(k \oplus M), D)$ by the left-hand degree and the $E_{1}$ term of the resulting spectral sequence has the form $\left(T(V) \otimes(k \oplus M), D_{2}\right)$ where $D_{2}=d_{2}$ on $T(V)$ and $\omega-\omega^{-1} d_{2} \omega$ on $T(V) \otimes M$; here $d_{2}$ denotes the quadratic part of the differential $D_{2}: V \rightarrow T^{2}(V)$. The map $\Psi$ defined above remains a quism when considered as a map

$$
\left(T(V) \otimes(k \oplus M), D_{2}\right) \stackrel{\Psi}{\rightarrow}\left(T(V) / T^{>m}(V), \bar{d}_{2}\right),
$$


as can easily be checked. We note for future reference the observation that $\operatorname{Ker} \Psi=T^{>m}(V) \oplus T(V) \otimes M$ is acyclic for $D$ and $D_{2}$ and that

$$
T(V) \otimes(k \oplus M)^{\geq m+p, *}=T^{\geq m+p}(V) \oplus T^{\geq p}(V) \otimes M .
$$

\section{Proof OF THE THEOREM}

To show that the $M-M$ s.s. collapses at the $(m+1)$ st stage, it suffices to show that for each $k \geq 0$, if $a \in T(V)$ is such that $d a \in T^{\geq m+k}(V)$ then there is an $\tilde{a} \in T^{\geq k}(V)$ with $d(a-\tilde{a})=0$. Note that for $k=0$ or 1 we may choose $\tilde{a}=a$. Now assume $k \geq 2$ and let $(T(V), d) \rightarrow\left(C^{*}(S, k), d\right)$ be a minimal free tensor model as above. With $\operatorname{Mcat}(S ; k)=m$, as in [FHLT, Step 6], this also guarantees a retraction $r:(T(V) \otimes(k \oplus M), D) \rightarrow(T(V), d)$.

Since $d a \in \operatorname{ker} \Psi$ is a cocycle, there is some $b \in \operatorname{ker} \Psi$ with $D b=d a$. Write $b=s b_{0}+b_{1}+b_{2}$ where $b_{0} \in T^{m+1}(V), b_{1} \in T^{\geq m+1}(V)$, and $b_{2} \in T^{\geq 1}(V) \otimes M$. A short computation shows that $D b-b_{0} \in[T(V) \otimes(k \oplus M)]^{\geq m+2, *}$. Since $D b=d a \in T^{\geq m+k}(V)$, this implies $b_{0}=0$. If $k=2$, set $\tilde{a}=r(b)$. Then $d(a-\tilde{a})=0$ and $r(b) \in T^{\geq 2}(V)$ since $m \geq 1, r(M) \subseteq T^{\geq 1}(V)$ and $r$ is a $T(V)$ module map.

If $k \geq 3$, write $b=b_{m+1}+b^{\prime}$ where the filtration degree of $b_{m+1}$ is $m+1$ and that of $b^{\prime}$ is at least $m+2$. Since $D b \in[T(V) \otimes(k \oplus M)]^{\geq m+k, *}$, we have $D_{2} b_{m+1}=0$. But $b_{m+1} \in \operatorname{ker} \Psi$ and so there is some $u_{m}$ of filtration degree $m$ with $D_{2} u_{m}=b_{m+1}$. Noting that $D-D_{2}$ increases filtration degree by at least 2 , we write $D b=D\left(D u_{m}-\left(D-D_{2}\right) u_{m}+b^{\prime}\right)=D(\tilde{b})$ where $\tilde{b}$ now has filtration degree at least $m+2$. As before, $r(\tilde{b}) \in T^{\geq 3}(V)$ and if $k=3$, we set $\tilde{a}=r(\tilde{b})$ and stop.

For $k \geq 4$, continue in this manner, using the acyclicity of $\operatorname{ker} \Psi$ with respect to $D_{2}$ until we get to $D(b)=D(\tilde{b})$ where $\tilde{b}$ has filtration degree at least $m+k-1$. Then $r(\tilde{b}) \in T^{\geq k}(V)$ and as before, we set $a^{\prime}=r(\tilde{b})$ to finish the proof.

\section{REFERENCES}

[FH] Y. Felix and S. Halperin, Rational L.-S. category and its applications, Trans. Amer. Math. Soc. 273 (1982), 1-37.

[FHLT] Y. Felix, S. Halperin, J. M. Lemaire, and J. C. Thomas, Mod p loop space homology, Inventiones Math. 95 (1989), 247-262.

[G] T. Ganea, Lusternik-Schnirelmann category and strong category, Illinois J. Math 11 (1967), 417-427.

[Gi] M. Ginsburg, On the L.S. category, Ann. of Math. 77 (1963), 538-551.

[He] K. Hess, A proof of Ganea's conjecture for rational spaces, Topology Vol. 30, No. 2, pp. 205-214, 1991.

[HL] S. Halperin and J.-M. Lemaire, Notions of category in differential algebra, Lecture Notes in Math., vol. 1318, Springer-Verlag, Berlin, 1988, pp. 138-154.

[S] P. A. Schweitzer, Secondary cohomology operations induced by the diagonal mapping, Topology 3 (1965), 337-355.

Department of Mathematics, University of Ottawa, 585 King Edward Avenue, OtTawa, Ontario, Canada

E-mail address: bjessup@acadvm1.uottawa.ca 